\title{
Exploring Disease Representation in Movies
}

\author{
Antonio Perciaccante, $M D^{7}$, Philippe Charlier, $M D^{2,3}$, Alessia Coralli, $B S N^{4}$, \\ Saudamini Deo, $P h D^{2}$, Otto Appenzeller, $\mathrm{MD}^{5,6}$, and Raffaella Bianucci, $P h D^{7,8,9}$
}

'Department of Medicine, San Giovanni di Dio Hospital, Gorizia, Italy; ${ }^{2}$ Section of Medical Anthropology (UVSQ, DANTE Laboratory EA 4498), UFR of Health Sciences, Montigny-Le-Bretonneux, France; ${ }^{3}$ Museum of Quai Branly - Jacques Chirac, Paris, France; ${ }^{4}$ AAS 2 Bassa Friulana - Isontina, Distretto Alto Isontino, Cormons, Italy; ${ }^{5} \mathrm{New}$ Mexico Health Enhancement and Marathon Clinics Research Foundation, Albuquerque, NM, USA; ${ }^{6} \mathrm{New}$ Mexico Museum of Natural History and Science, Albuquerque, NM, USA; ${ }^{7}$ Legal Medicine Section, Department of Public Health and Paediatric Sciences, University of Turin, Turin, Italy; ${ }^{8}$ Warwick Medical School, Microbiology and Infection Division, University of Warwick, Warwick, UK; ${ }^{9}$ UMR 7268, Laboratoire d'Anthropologie bio-culturelle, Droit, Etique \& Santé (Adés), Faculté de Médecine de Marseille, Marseille, France.

J Gen Intern Med 34(11):2351-4

DOI: $10.1007 / \mathrm{s} 11606-019-05254-6$

(c) Society of General Internal Medicine 2019

$\mathrm{T}$ he Academy Awards, also known as Oscars, are given annually by the Academy of Motion Picture Arts and Sciences. To celebrate the 90 years of this prestigious prize, and to highlight the relationship between cinematography and medicine, we analyzed the report of 554 films, which received nominations to "Oscar for Best Picture" since the first edition in $1929 .{ }^{1}$

To perform an accurate analysis, three authors independently evaluated the movies' plots to searching references to illnesses or other aspects related to the medicine. The cases of disagreement have been discussed with a group of further three authors.

War wounds excluded, we found that among the nominated films, medicine references are reported in the plot of 105 (18.9\%) (Table 1). Medicine references are present in twenty (21.9\%) of ninety-one awarded films. The distribution of films referring to medicine is reported in Figure 1.

Our analysis shows that the most represented diseases are the psychiatric (21.9\%) ones followed by neurological ones (13.3\%), alcohol addiction (13.3\%), and infectious diseases (13.3\%).

Psychiatric diseases continue to have significant emotional and social impact but the methods of their treatments have always been a topic of ethical debate. It is interesting to note the references to passive-aggressive behavior, anxiety, delirium, and the use of questionably ethical treatment such as electro-shock therapy and lobotomy in "One Flew Over the Cuckoo's Nest."

Interesting are also the depictions of psychiatric diseases, such as schizophrenia, depression, post-traumatic stress, obsessive-compulsive, and bipolar disorders. Significant mental disabilities were brought to public attention by the movie "Rain Man," in which the protagonist suffered from Savant syndrome.

A strong medical and social impact have also alcohol and drug addictions; the last are prevalent in films from the 2000s onwards.

Published online August 13, 2019
Frequently reported illnesses are neurological diseases, which affect considerably the quality of life of the patients and their relatives. There have been references to ischemic stroke, senile dementia, cerebral hemorrhage, epidemic encephalitis, cerebral palsy, post-traumatic paraparesis, and amyotrophic lateral sclerosis as reported in biopics on Lou Gehrig and Stephen Hawking.

References to infectious diseases (scarlet, yellow, typhoid fevers, bubonic plague, measles, pneumonia) occur mostly in the movies of the 1930s and 1940s, when they were the most common causes of mortality. Moreover, five films set at the beginning of the twentieth century refer to tuberculosis, whereas HIV infection is reported mostly in movies set in the 1980s, when the disease was at its peak and highly stigmatized.

Surprisingly, two of the most widespread diseases of twentieth and twenty-first centuries are under-represented: we found nine references to oncological diseases, and only three references to heart diseases (respectively only $8.5 \%$ and $2.8 \%$ of the films referring to medicine).

We further report references to mutism and deaf-mutism, amnesia, insomnia, hemophilia, carbon monoxide poisoning, and two rare diseases such as the Proteus syndrome and the picnodysostosis, in "The Elephant Man" and in "Moulin Rouge," respectively. Only few references can be found to metabolic diseases such as gout and diabetes mellitus.

From the 1990s onwards, some movies deal with important themes, such as euthanasia, clandestine abortion, medical malpractices, and relationship between environmental pollution and cancer.

Lastly, we wish to underline the plots of some films dealing with medicine and ethics in medicine; the biopic "The Story of Louis Pasteur" describes Pasteur's discoveries; "The Citadel," a film, in which the protagonist, a Scottish doctor, initially dedicated to cure miners affected by tuberculosis changes his mind after a meeting with a colleague and decides to solely serve the rich hypochondriacs.

Our analysis confirms that medicine and disease are an intrinsic part of humanity and, hence, serve as quasi-infinite source of inspiration for film-makers of past and present alike. 
Table 1 Films with Medical References Received Nomination for "Oscar for Best Picture"

\begin{tabular}{|c|c|c|c|c|c|}
\hline $\begin{array}{l}\text { Year of } \\
\text { nomination }\end{array}$ & Winner & Title & Director & Cast & Medical references \\
\hline 1930 & Not & The Patriot & Ernst Lubitsch & Emil Jannings & Paranoid personality \\
\hline 1932 & Not & Arrowsmith & John Ford & Ronald Colman & Bubonic plague \\
\hline 1934 & Not & Little Women & George Cuckor & Katharine Hepburn & Scarlet fever \\
\hline 1935 & Not & $\begin{array}{l}\text { The Barretts of Wimpole } \\
\text { street }\end{array}$ & Sidney Franklin & $\begin{array}{l}\text { Norma Shearer } \\
\text { Fredric March }\end{array}$ & Undisclosed illness \\
\hline 1936 & Not & Captain Blood & Michael Curtiz & Errol Flynn & Gout \\
\hline 1937 & Not & The Story of Luis Pasteur & William Dieterle & Paul Muni & Asepsis, vaccinations \\
\hline 1938 & Yes & The Life of Emile Zola & William Dieterle & Paul Muni & Carbon monoxide poisoning \\
\hline 1939 & Not & The Citadel & King Vidor & Robert Donation & Medical ethics \\
\hline 1939 & Not & Jezebel & William Wyler & $\begin{array}{l}\text { Bette Davis } \\
\text { Hanry Fonda }\end{array}$ & Yellow fever \\
\hline 1940 & Yes & Gone with the Wind & Victor Fleming & $\begin{array}{l}\text { Clark Gable } \\
\text { Vivien Leigh }\end{array}$ & $\begin{array}{l}\text { Measles } \\
\text { Pneumonia } \\
\text { Typhoid fever }\end{array}$ \\
\hline 1940 & Not & Dark Victory & Edmund Goulding & Bette Davis & Brain tumor \\
\hline 1942 & Not & The Little Foxes & William Wyler & $\begin{array}{l}\text { Bette Davis } \\
\text { Herbert Marshall }\end{array}$ & Heart attack \\
\hline 1943 & Not & The Pride of the Yankees & Sam Wood & Gary Cooper & Amyotrophic lateral sclerosis \\
\hline 1943 & Not & Random Harvest & Mervyn LeRoy & Ronald Colman & Post-traumatic amnesia \\
\hline 1943 & Not & The Magnificent Ambersons & Orson Welles & Joseph Cotten & Tuberculosis \\
\hline 1943 & Not & Kings Row & Sam Wood & $\begin{array}{l}\text { Ann Sheridan } \\
\text { Robert Cummings } \\
\text { Ronald Reagan }\end{array}$ & $\begin{array}{l}\text { Ethics and medicine } \\
\text { Unnecessary legs amputation }\end{array}$ \\
\hline 1944 & Not & The Song of Bernadette & Henry King & Jennifer Jones & Tuberculosis \\
\hline 1946 & Yes & The Lost Weekend & Bill Wilder & Ray Milland & $\begin{array}{l}\text { Alcoholism } \\
\text { Depression }\end{array}$ \\
\hline 1946 & Not & Spellbound & Alfred Hitchcock & $\begin{array}{l}\text { Ingrid Bergman } \\
\text { Gregory Peck }\end{array}$ & Amnesias \\
\hline 1946 & Not & Mildred Pierce & Michael Curtiz & $\begin{array}{l}\text { Joan Crawford } \\
\text { Jack Carson }\end{array}$ & Pneumonia \\
\hline 1946 & Not & The Bells of St. Mary's & Leo McCarey & $\begin{array}{l}\text { Bing Crosby } \\
\text { Ingrid Bergman }\end{array}$ & Tuberculosis \\
\hline 1947 & Not & The Razor's Edge & Edmund Goulding & $\begin{array}{l}\text { Tyrone Power } \\
\text { Gene Tierney }\end{array}$ & Alcohol addiction \\
\hline 1947 & Not & The Yearling & Clarence Brown & $\begin{array}{l}\text { Gregory Peck } \\
\text { Jane Wyman }\end{array}$ & Unspecified neonatal deaths \\
\hline 1948 & Not & Johnny Belinda & Jean Negulesco & $\begin{array}{l}\text { Jane Wyman } \\
\text { Lew Ayres }\end{array}$ & Deaf-mutism \\
\hline 1949 & Not & The Snake Pit & Anatole Litvak & Olivia de Havilland & Schizophrenia \\
\hline 1952 & Not & A Streetcar Named Desire & Elia Kazan & $\begin{array}{l}\text { Vivien Leigh } \\
\text { Marlon Brando }\end{array}$ & Alcohol addiction \\
\hline 1953 & Yes & The Greatest Show on Earth & Cecil B. DeMille & $\begin{array}{l}\text { Betty Hutton } \\
\text { Cornel Wilde }\end{array}$ & Post-injury harm paralysis \\
\hline 1953 & Not & Moulin Rouge & John Huston & Josè Ferrer & Picnodysostosis \\
\hline 1955 & Not & The Country Girl & George Seaton & $\begin{array}{l}\text { Grace Kelly } \\
\text { Bing Crosby }\end{array}$ & Alcohol addiction \\
\hline 1955 & Not & The Caine Mutiny & Edward Dmytryk & $\begin{array}{l}\text { Humphrey Bogart } \\
\text { Josè Ferrer }\end{array}$ & $\begin{array}{l}\text { Panic attacks } \\
\text { Paranoid personality }\end{array}$ \\
\hline 1958 & Not & Peyton Place & Marco Robson & $\begin{array}{l}\text { Lana Turner } \\
\text { Hope Lange }\end{array}$ & Alcohol addiction \\
\hline 1959 & Not & Cat on a Hot Tin Roof & Richard Brooks & $\begin{array}{l}\text { Elizabeth Taylor } \\
\text { Paul Newman }\end{array}$ & $\begin{array}{l}\text { Alcoholism } \\
\text { Cancer }\end{array}$ \\
\hline 1961 & Not & Sons and Lovers & Jack Cardiff & $\begin{array}{l}\text { Trevor Howard } \\
\text { Dean Stockwell } \\
\text { Wendy Hiller }\end{array}$ & $\begin{array}{l}\text { Oedipus complex } \\
\text { Pneumonia } \\
\text { Cancer }\end{array}$ \\
\hline 1961 & Not & Elmer Granty & Richard Brooks & Burt Lancaster & Alcoholism \\
\hline 1966 & Not & Ship of Fools & Stanley Kramer & $\begin{array}{l}\text { Vivien Leigh } \\
\text { Simone Signoret }\end{array}$ & Opioid addiction \\
\hline 1966 & Not & Doctor Zhivago & David Lean & Omar Sharif & Myocardial infarction \\
\hline 1967 & Not & $\begin{array}{l}\text { Who's Afraid of Virginia } \\
\text { Woolf? }\end{array}$ & Mike Nichols & $\begin{array}{l}\text { Elizabeth Taylor } \\
\text { Richard Burton }\end{array}$ & Alcohol addiction \\
\hline 1970 & Not & $\mathrm{Z}$ & Costa Gravas & $\begin{array}{l}\text { Yves Montand } \\
\text { Irene Papas }\end{array}$ & Post-traumatic cerebral hemorrhage \\
\hline 1971 & Not & Love story & Arthur Hiller & Ali MacGraw & Leukemia \\
\hline 1972 & Not & Nicholas and Alexandra & Franklin J Schaffner & $\begin{array}{l}\text { Michael Jayston } \\
\text { Janet Suzman }\end{array}$ & Hemophilia \\
\hline 1974 & Not & Cries and Whispers & Ingmar Bergman & Harriet Andersson & Uterine cancer \\
\hline 1975 & Not & The Conversation & Francis Ford Coppola & Gene Hackman & Obsessive-compulsive disorder \\
\hline 1975 & Not & Lenny & Melvin Frank & Dustin Hoffman & Alcohol and drug addiction \\
\hline 1976 & Not & Dog Day Afternoon & Sidney Lumet & Al Pacino & Diabetes mellitus \\
\hline 1976 & Yes & $\begin{array}{l}\text { One Flew Over the Cuckoo's } \\
\text { Nest }\end{array}$ & Miloš Forman & Jack Nicholson & $\begin{array}{l}\text { Passive-aggressive behavior } \\
\text { Anxiety } \\
\text { Delirium }\end{array}$ \\
\hline
\end{tabular}


Table 1. (continued)

\begin{tabular}{|c|c|c|c|c|c|}
\hline $\begin{array}{l}\text { Year of } \\
\text { nomination }\end{array}$ & Winner & Title & Director & Cast & Medical references \\
\hline & & & & & Epilepsy \\
\hline 1977 & Not & Taxi Driver & Martin Scorsese & Robert De Niro & Chronic insomnia \\
\hline 1978 & Yes & Annie Hall & Woody Allen & Woody Allen & Necroses \\
\hline 1979 & Yes & The Deer Hunter & Micheal Cimino & Robert De Niro & Post-traumatic stress disorder \\
\hline 1980 & Not & All That Jazz & Bob Fosse & $\begin{array}{l}\text { Roy Scheider } \\
\text { Jessica Lange }\end{array}$ & Coronary artery disease \\
\hline 1981 & Yes & Ordinary People & Robert Redford & $\begin{array}{l}\text { Donald Sutherland } \\
\text { Timothy Hutton }\end{array}$ & Post-traumatic stress disorder \\
\hline 1981 & Not & The Elephant Man & David Lynch & $\begin{array}{l}\text { John Hurt } \\
\text { Anthony Hopkins }\end{array}$ & Protheus syndrome \\
\hline 1982 & Not & On Golden Pond & Mark Rydell & $\begin{array}{l}\text { Katharine Hepburn } \\
\text { Henry Fonda }\end{array}$ & Amnesias \\
\hline 1983 & Not & The Verdict & Sidney Lumet & Paul Newman & Medical malpractice \\
\hline 1983 & Not & Tootsie & Sydney Pollack & $\begin{array}{l}\text { Dustin Hoffman } \\
\text { Jessica Lange }\end{array}$ & Depression \\
\hline 1984 & Yes & Terms of Endearment & James L. Brooks & $\begin{array}{l}\text { Shirley MacLaine } \\
\text { Debra Winger }\end{array}$ & Cancer \\
\hline 1984 & Not & Tender Mercies & Bruce Beresford & $\begin{array}{l}\text { Robert Duvall } \\
\text { Tess Harper }\end{array}$ & Alcohol addiction \\
\hline 1985 & Yes & Amadeus & Milos Forman & $\begin{array}{l}\text { Tom Hulce } \\
\text { F. Murray Abraham }\end{array}$ & $\begin{array}{l}\text { Depression } \\
\text { Alcohol addiction }\end{array}$ \\
\hline 1986 & Yes & Out of Africa & Sydney Pollack & $\begin{array}{l}\text { Meryl Streep } \\
\text { Robert Redford }\end{array}$ & Syphilis \\
\hline 1987 & Not & Children of a Lesser God & Randa Haines & $\begin{array}{l}\text { William Hurt } \\
\text { Marlee Matlin }\end{array}$ & Deaf-mutism \\
\hline 1989 & Yes & Rain Man & Barry Levinson & $\begin{array}{l}\text { Dustin Hoffman } \\
\text { Tom Cruise }\end{array}$ & Savant syndrome \\
\hline 1990 & Yes & Driving Miss Daisy & Bruce Beresford & $\begin{array}{l}\text { Jessica Tendy } \\
\text { Morgan Freeman }\end{array}$ & Senile dementia \\
\hline 1990 & Not & Born on the Fourth of July & Oliver Stone & Tom Cruise & $\begin{array}{l}\text { Post-traumatic paraparesis } \\
\text { Alcohol addiction }\end{array}$ \\
\hline 1990 & Not & My Left Foot & Jim Sheridan & Daniel Day Lewis & Cerebral palsy $\ldots$ \\
\hline 1991 & Not & Awakenings & Penny Marshall & $\begin{array}{l}\text { Robert De Niro } \\
\text { Robin Williams }\end{array}$ & Letargia due to epidemic encephalitis \\
\hline 1992 & Not & The Prince of Tides & Barbra Streisand & $\begin{array}{l}\text { Nick Nolte } \\
\text { Barbra Streisand }\end{array}$ & $\begin{array}{l}\text { Chronic depression } \\
\text { Dissociative state }\end{array}$ \\
\hline 1994 & Not & The Piano & Jean Campion & Holly Hunter & Mutism \\
\hline 1995 & Yes & Forrest Gump & Robert Zemeckis & Tom Hanks & $\begin{array}{l}\text { Curve spine } \\
\text { Marginal intelligence }\end{array}$ \\
\hline 1995 & Not & Four Weddings and a Funeral & Mike Newell & Hugh Grant & Deaf-mutism \\
\hline 1997 & Yes & The English Patient & Anthony Mingella & $\begin{array}{l}\text { Ralph Fiennes } \\
\text { Juliette Binoche }\end{array}$ & Euthanasia \\
\hline 1997 & Not & Shine & Scott Hicks & Geoffrey Rush & $\begin{array}{l}\text { Manic syndrome } \\
\text { Schizophrenia }\end{array}$ \\
\hline 1998 & Not & As Good as It Gets & James L. Brooks & $\begin{array}{l}\text { Jack Nicholson } \\
\text { Helen Hunt }\end{array}$ & Obsessive-compulsive disorder \\
\hline 2000 & Not & The Sixth Sense & M. Night Shyamalan & Bruce Willis & Munchausen syndrome \\
\hline 2000 & Not & The Green Mile & Frank Darabont & $\begin{array}{l}\text { Tom Hanks } \\
\text { David Morse }\end{array}$ & $\begin{array}{l}\text { Bladder infection } \\
\text { Brain tumor }\end{array}$ \\
\hline 2000 & Not & The Cider House Rules & Lasse Hallström & $\begin{array}{l}\text { Tobey Maguire } \\
\text { Charlize Theron }\end{array}$ & Clandestine abortion \\
\hline 2001 & Not & Chocolat & Lasse Hallström & $\begin{array}{l}\text { Juliette Binoche } \\
\text { Johnny Depp }\end{array}$ & Diabetes mellitus \\
\hline 2001 & Not & Erin Brockovich & Steven Soderbergh & Julia Roberts & $\begin{array}{l}\text { Environmental pollution and risk of } \\
\text { cancer }\end{array}$ \\
\hline 2002 & Yes & A Beautiful Mind & Ron Howard & Russell Crowe & Schizophrenia \\
\hline 2002 & Not & Moulin Rouge! & Baz Luhrmann & Nicole Kidman & Tuberculosis \\
\hline 2003 & Not & The Mours & Stephen Daldry & Nicole Kidman & $\begin{array}{l}\text { Bipolar disorder } \\
\text { Uterine fibroma }\end{array}$ \\
\hline 2005 & Yes & Million Dollar Baby & Clint Eastwood & $\begin{array}{l}\text { Clint Eastwood } \\
\text { Hilary Swank }\end{array}$ & $\begin{array}{l}\text { Post-traumatic paralysis } \\
\text { Euthanasia }\end{array}$ \\
\hline 2005 & Not & Finding Neverland & Marc Forster & $\begin{array}{l}\text { Johnny Depp } \\
\text { Kate Winslet }\end{array}$ & Tuberculosis/lung cancer \\
\hline 2005 & Not & The Aviator & Martin Scorsese & Leonardo Di Caprio & Obsessive-compulsive disorder \\
\hline 2005 & Not & Ray & Taylor Hackford & Jamie Foxx & $\begin{array}{l}\text { Blindness } \\
\text { Opioid addiction }\end{array}$ \\
\hline 2007 & Not & Babel & $\begin{array}{l}\text { Alejandro González } \\
\text { Iñárritu }\end{array}$ & $\begin{array}{l}\text { Brad Pitt } \\
\text { Cate Blanchet } \\
\text { Koji Yakusho }\end{array}$ & Deaf-mutism \\
\hline 2007 & Not & Little Miss Sunshine & $\begin{array}{l}\text { Jonathan Dayton } \\
\text { Valerie Faris }\end{array}$ & $\begin{array}{l}\text { Greg Kinnear } \\
\text { Toni Collette } \\
\text { Alan Arkin }\end{array}$ & Opioid addiction \\
\hline 2010 & Not & Precious & Lee Daniels & Gabourey Sidibe & Obesity \\
\hline
\end{tabular}


Table 1. (continued)

\begin{tabular}{|c|c|c|c|c|c|}
\hline $\begin{array}{l}\text { Year of } \\
\text { nomination }\end{array}$ & Winner & Title & Director & Cast & Medical references \\
\hline & & & & & $\begin{array}{l}\text { HIV } \\
\text { Down syndrome }\end{array}$ \\
\hline 2011 & Yes & The King's Speech & Tom Hooper & $\begin{array}{l}\text { Colin Firth } \\
\text { Geoffrey Rush }\end{array}$ & Stutter \\
\hline 2011 & Not & Black Swan & Darren Aronofsky & $\begin{array}{l}\text { Natalie Portman } \\
\text { Vincent Cassel }\end{array}$ & Self-harm \\
\hline 2011 & Not & The Fighter & David O. Russell & $\begin{array}{l}\text { Mark Wahlberg } \\
\text { Christian Bale }\end{array}$ & Drug addiction \\
\hline 2011 & Not & Winter's Bone & Debra Granik & Jennifer Lawrence & Depression \\
\hline 2012 & Not & The Descendants & Alexander Payne & George Clooney & Coma \\
\hline 2013 & Not & Silver Lining Playbook & David O. Russell & Bradley Cooper & Bipolar disorder \\
\hline 2013 & Not & Beasts of the Southern Wild & Benh Zeitlin & $\begin{array}{l}\text { Quvenzhané Wallis } \\
\text { Dwight Henry }\end{array}$ & Undefined heart disease \\
\hline 2013 & Not & Amour & Michael Haneke & $\begin{array}{l}\text { Jean Louis } \\
\text { Trintignant } \\
\text { Emmanuelle Riva }\end{array}$ & $\begin{array}{l}\text { Stroke } \\
\text { Carotid artery stenosis }\end{array}$ \\
\hline 2014 & Not & Dallas Buyers Club & Jean Marc Vallée & $\begin{array}{l}\text { Matthew } \\
\text { McConaughey }\end{array}$ & $\begin{array}{l}\text { HIV } \\
\text { AIDS }\end{array}$ \\
\hline 2014 & Not & The Theory of Everything & James Marsh & Eddie Redmaine & Amyotrophic lateral sclerosis \\
\hline 2014 & Not & The Wolf of Wall Street & Martin Scorsese & Leonardo Di Caprio & Drug addiction \\
\hline 2014 & Not & Philomena & Stephen Frears & $\begin{array}{l}\text { Judi Dench } \\
\text { Steve Coogan }\end{array}$ & AIDS \\
\hline 2014 & Not & Nebraska & Alexander Payne & $\begin{array}{l}\text { Bruce Dern } \\
\text { Will Forte }\end{array}$ & Alcohol addiction \\
\hline 2017 & Yes & Moonlight & Barry Jenkins & $\begin{array}{l}\text { Trevante Rhodes } \\
\text { Janelle Monàe }\end{array}$ & Opioid addiction \\
\hline 2017 & Not & Manchester by the Sea & Kenneth Lonergan & $\begin{array}{l}\text { Casey Affleck } \\
\text { Michelle Williams }\end{array}$ & Alcohol addiction \\
\hline 2018 & Yes & The Shape of Water & Guillermo del Toro & Sally Hawkins & Mutism \\
\hline 2018 & Not & $\begin{array}{l}\text { Three Billboards Outside } \\
\text { Ebbing, Missouri }\end{array}$ & Martin McDonagh & $\begin{array}{l}\text { Frances McDormand } \\
\text { Woody Harrelson }\end{array}$ & Pancreatic cancer \\
\hline 2018 & Not & Phantom Thread & Paul Thomas Anderson & $\begin{array}{l}\text { Daniel Day Lewis } \\
\text { Vicky Krieps }\end{array}$ & Mushroom poisoning \\
\hline 2019 & Not & A Star is Born & Bradley Cooper & $\begin{array}{l}\text { Bradley Cooper } \\
\text { Lady Gaga }\end{array}$ & Tinnitus \\
\hline
\end{tabular}

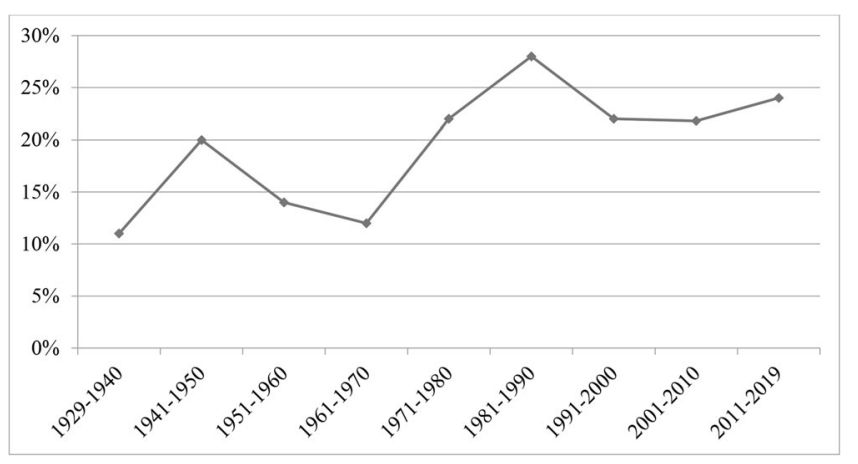

Figure 1 Percentage of films with reference to medicine during the different decades. The figure shows the percentage of films referring to medicine, calculated as: (number films with medicine references number films which received nomination to "Oscar for Best Picture") $\times 100$.
Corresponding Author: Antonio Perciaccante, MD; Department of Medicine, San Giovanni di Dio Hospital, Gorizia, Italy (e-mail: antonioperciaccante@libero.it).

\section{Compliance with Ethical Standards:}

Conflict of Interest: The authors declare that they do not have a conflict of interest.

\section{REFERENCES}

1. Academy Awards. Official website http://awardsdatabase.oscars.org/ search/results. Accessed March 01, 2019.

Publisher's Note Springer Nature remains neutral with regard to jurisdictional claims in published maps and institutional affiliations. 